\title{
Does the immune system of plant and animal kingdoms share any pathways or mechanisms of action in phytotherapy?
}

\begin{abstract}
Phytotherapy, herbal remedies and dietary supplementation are recovering, to the present day, more and more attention from the scientific world of the Western countries not only in virtue of the antibiotic-resistance emerging problem or of the hypersensitivity towards some synthetic molecules typical of the conventional medicine, but also because the attention of the consumer/patient is increasingly oriented towards the respect for the environment and trusts the green economy as the only sustainable policy. However, even if various herbal remedies are recognized as antioxidant, antibacterial, anticancer and immunostimulant, the known functioning mechanisms of phytocomplexes are still scarse. Furthermore, the in vitro evaluation of the mechanisms of action of the single main components are frequently not able to reproduce the general effect linked to the intake of the phytocomplex in it's entirely. In this article, hypothesizing that the key of the effectiveness of phytotherapy is due to the triggering of common pathways existing between the innate immune system of the plant and animal kingdom, it is wanted to try to give a general explanation to the functioning principle of phytotherapy in the animal world. In light of this theory, so as the route of administration of many vaccines (oral vs injection) has changed in the last few years due to the improved knowledge on the operting of mucosal immune system, similarly the therapeutic approach of some diseases could also be reconsidered.
\end{abstract}

Volume 6 Issue 4 - 2018

\author{
Daniela Beghelli \\ School of Biosciences and Veterinary Medicine, University of \\ Camerino, Italy
}

Correspondence: Daniela Beghelli, School of Biosciences and Veterinary Medicine, University of Camerino, Via Gentile III da Varano, 62032 Camerino (MC) Italy, Tel: +39 737 40 320I, E-mail daniela.beghelli@unicam.it

Received: July 14, 2018 | Published: August 13, 2018

Keywords: plant immune system, mucosal immunity, phytotherapy, innate immunity, animal immune system, phytocomplexes, regulatory molecules, humoral

\section{Introduction}

The question arises from the need to understand why the use of phytotherapy may modulate the immune responses even in the animal kingdom as reported by some authors. ${ }^{1}$ In fact, even if the traditional use of herbal medicine products may guarantee efficacy, for very few medical plants scientific data on mechanisms of action are available. ${ }^{2}$ The term Phytotherapy, derived from the Greek words 'Phyto' and 'therapy', was introduced into science by the French physician Henri Leclerc (1870-1955) and indicates the therapy practiced with medicaments of vegetable origin. A study conducted by the World Health Organization had reported that about $80 \%$ of world's population relies on traditional medicine. The history of phytotherapy is very old and was presumably one of the first therapeutic methods undertaken by man. Already in ancient times (since the Egyptian and Mesopotamian era), mankind was so fascinated by the therapeutic action of plants that for centuries magical and divine properties were attributed to them. Later on, humans have learnt by experience and observations how to use plants correctly and since the nineteenth century the empirical use of plants has been brought back within the boundaries of rationality and scientific rigor. But in what way has man been using these plants for millenia? These 'preparations' of vegetable origin have always been used through essentially three administration routes: at a lesser extent, by local applications or fumigation, otherwise mainly by ingestion. The administration of herbal medicine products through the oral route may represent a crucial point, as we will discuss below, to explain the efficacy of the traditional medicine. Therefore, the text found in the work On Aliment: "In food excellent medication, in food bad medication, bad and good relatively", 3 nowadays attributed to the Hellenistic period, but in Antiquity (by Galenus in particular) erroneously associated with Hippocrates, brings us back to why mankind at some point has started to ingest plants or their fruits, roots and leaves in order to find in them not only nourishment and gratification, but also a therapeutic remedy for its illnesses. Indeed, the idea of using plants as medicine treatment was probably born from fortuitous observations or from the experiences that many plants used in nutrition could also prove to be toxic or poisonous or, better, able to improve disorders. However, also Hippocrates from Cos (around $460 \mathrm{BC}$-around $375 \mathrm{BC}$ ), the father of Western modern medicine, knowing that food was closely linked to health and disease, applied dietetic measures for the benefit of the sick. ${ }^{4}$ According to the World Health Organization (WHO), every vegetable that contains, in one or more of its organs, pharmacologically active substances deserves the name of a medicinal plant. The pharmacognosy studies have evidenced that the set of these pharmacologically active molecules, called phytocomplexes, have the ability to work in synergy with all components. A phytocomplex represents the integral pharmacological unit of a medical plant. Most of the natural phytocomplexes that exert therapeutic actions, once ingested, have shown to act as antioxidants (thus reducing the levels of free radicals), as anti-inflammatory molecules (thus reducing the risk of chronic inflammatory diseases), as anticancer, antimicrobial and immune modulators. ${ }^{1}$ Living organisms such as plants and animals can be considered as a laboratory of biosynthesis that must provide not only for their own needs but also for their own defense. The afore mentioned phytocomplexes may, therefore, represent the set of molecules developed even to protect the plant life itself. Indeed, plants as well as animal beings are constantly attacked by environmental pathogens that through their entry into these living organisms look for their survival in turn. 
This struggle for survival permitted a refinement of the means for defense and attack both in the plant and in the animal kingdom. Is it then possible that the molecular systems that the plant has developed for its preservation also elicited immune responses in the animal kingdom once 'the latter' had ingested/absorbed this set of molecules? And if so, which kind of immune responses are they able to give raise to?

\section{Vertebrate immune system}

Vertebrate immune defense is one of the most complex biological phenomena. It consists of two components: the innate and the acquired immune systems (humoral and cellular immunity) that induce both the systemic and the mucosal immune responses. ${ }^{5-6}$ Furthermore, the immune system may have a large reserve capacity ${ }^{7}$ and may reconfigure itself or shift from one type to another to optimize its response to different conditions. ${ }^{8}$ At the gut mucosal level, the innate immune response not only represents the first line of defense against pathogenic microorganisms, but provides also immune-tolerance and the biological signals that instruct the adaptive immune system to unleash a response. ${ }^{5-9}$ Importantly, it happens also at the presence of non-commensal and probiotic bacteria ${ }^{10}$ or of herbal medicine as well. ${ }^{11}$ The common mucosal immune system in vertebrates (comprising the gut-, bronchial- and nasal-associated lymphatic tissues) is unique insofar as it can provide both positive and negative signals not only for the induction but also regulation of immune responses in both the mucosal and systemic compartments after oral or nasal antigen challenge. ${ }^{12-13}$ Between its defense tools against pathogens, the mucosal innate immunity harbors a series of physical, chemical and biological innate molecules (i.e. antimicrobial peptides) that cooperate with the innate lymphoid cells (ILCs). The last, although shares numerous similarities with $\mathrm{CD} 4+$ and CD8+ $\mathrm{T}$ cells, can respond to various stimuli independently of major histocompatibility-dependent interactions. ${ }^{14}$ On the contrary, the mucosal adaptive system comprises various antigen-presenting cells, like dendritic cells (that cross talk with both innate and adaptive systems) and T and B lymphocytes. ${ }^{15}$ However, in mucosal innate immunity remarkable importance has also to be attributed to receptors like Toll-like receptors (TLRs), NOD-like receptors, and to RIGI-like receptors. The evolutionarily conserved Toll-like receptors have been shown to be essential receptors, capable of recognizing the highly conserved structural cytosolic pathogen-associated microbial patterns (PAMPs) with their common pathogen-associated molecules such as lipopolysaccharide and peptidoglycan. ${ }^{16}$ TLRs are differently distributed on almost all mucosal antigen presenting cells (macrophages and dendritic cells), B cells, follicle-associated epithelium, and $\mathrm{M}$ cells ${ }^{17}$ and, once activated, their signaling pathway leads to the expression of numerous genes, activation of transcription factors and synthesis of pro-inflammatory and effector cytokines that direct the adaptive immune response. Therefore, the process of TLRmediated PAMP recognition and the subsequent signaling cascade are thought to bridge innate and acquired immunity in the creation of protective immune responses against environmental agents. Some authors ${ }^{15}$ hypothesized that at the mucosal level the innate immunity is the true regulator of the immune system. Indeed, the mucosal immune system is constantly exposed to external environment comprising the resident, highly complex mucosal microbiota, antigens of food origin or from inhaled air, environmental xenobiotics, and potential pathogens and their products. These factors, all together, represent the primary force that has driven the development and stimulation of the entire immune system during evolution as well as in ontogeny. The evolutionary selective pressure of these environmental antigens has resulted in the strategic distribution throughout the mucosae of cells involved in the uptake, processing, and presentation of antigens and in the production of humoral and cellular factors of innate and specific immunity. ${ }^{18}$ Each pattern recognition receptor in innate mucosal immunity has the capability to distinguish PAMPs and, unlike the adaptive response, it is genetically and evolutionarily conserved, highly specialized and heritable, thus making mucosal immunity the primary line of defense in almost all mammalian body systems.

\section{Plant immune system}

Plants also have a sophisticated immune system that protects them from different kinds of pathogen; however, since they lack circulating cells, they rely on innate immunity and on systemic signals emanating from infection site. ${ }^{19}$

In this Kingdom, the first line of defense is represented by the cell wall with its enzymes, oligosaccharides and endomembrane system. Endocytic membrane trafficking from the plasma membrane (PM) to the vacuole is an important mechanism that allows the plant to respond to environmental changes and to subsequently reinstate the homeostatic condition of the cells. Furthermore, in the interface between a host cell and a microbial pathogen, could also be present unusual membrane structures called extracellular vesicles (EVs), whose functions are still to be elucidated. These exosomes could play a role in the direct delivery of antimicrobial compounds, or deliver RNA molecules that modulate defense signaling pathways or regulate cell-to-cell communication..$^{20}$ Finally, distributed at PM level, there are receptors, named transmembrane pattern recognition receptors (PRRs) that have a 'function TLRs-like' (in animals) and that recognize pathogen associated molecular patterns (PAMPs), such as bacterial flagellin or fungal chitin or danger associated molecular patterns (DAMPs). This type of immune response is called PAMPtriggered immunity $(\mathrm{PTI})^{19,20}$ and frequently it is strong enough against most pathogens to avoid infection. Once there has been the binding between PAMPs/DAMPs and PRRs, the plant immune processes continue with the endocytosis of the pattern recognition receptors and their intracellular trafficking up till destruction. However, when some highly adapted pathogens escape this immune response by injecting or secreting effector proteins that interfere with the PTI response in host cells, ${ }^{21}$ plants are able to use others immune receptors like nucleotide binding domain leucine-rich repeat (NB-LRR) to recognize effectors and activate a second level of immune response: the effector-triggered immunity (ETI) that acts largely inside the cell ${ }^{19}$.The PTI and ETI pathways share many signaling components (i.e. same co-factors) and the difference between these two lies in the ETI's greater intensity of response which often results in the hypersensitive responsetype of programmed cell death (HR-PCD) that physically isolates the infection. The latter is also indirectly activated by chloroplasts (organelles existing inside the cells) that, during ETI and PTI, produce defense signals such as ROS and salicylic acid that induce a downstream signaling cascade that activates HR-PCD. ${ }^{20}$ Pathogen effectors from diverse kingdoms are recognized by NB-LRR proteins, and activate similar defense responses. Interestingly, plant NB-LRR proteins are broadly related to animal CATERPILLER/ NOD/NLR proteins ${ }^{19}$ that are highly conserved through evolution. In cell plants, nanodomains regulate the different mechanisms that maintain the signaling specificity and specific defense responses of these shared signaling pathways. Since intracellular compartments have distinct biological functions in plants, plant cellular homeostasis 
really depends on the regulation of phytochemicals and subcellular environment. Subcellular compartments, endomembrane, intracellular trafficking, and communications of intracellular organelles (i.e. chloroplasts, stromules) play an important role not only in responding to environmental changes (and subsequently in reinstating the homeostatic condition of the cells), but also in the modulation of physiological changes during immunity. Studies have shown that the aforementioned pathogen effectors target just the host proteins that function in diverse plant biological processes of various subcellular compartments to suppress the pathways involved in immune responses. ${ }^{20}$ However, detection of PAMPs and effectors by the plant leads nucleus of the host cell infected to reprogramming of the transcriptome to initiate defense responses. Unconventional, non-degradative ubiquination has been discovered to play a critical role in the regulation/modulation of plant immunity. Ubiquination is known to be one of the most abundant protein modification processes (protein degradation, but also non-degradative/regulatory process) that occurs in the cells; it is highly diverse and complex and, between the many types of protein post-translational modification, it is unique to eukaryotes. ${ }^{22}$ Accordingly, a distinct fate for proteins involved in different cellular function or their subcellular distribution can be regulated through different types of ubiquitin modification. Furthermore, ubiquitination can also be reversed, adding more complexity to the fate of proteins. To date, ubiquination has been shown to regulate nearly all aspects of plant biology, ${ }^{23}$ including immune signaling. It is noteworthy that between the large number of ubiquitin enzymes (reflecting their role as the major governing factors for appropriate selection of a myriad of cellular substrate proteins to be modified by ubiquitin), the role of recently identified RBR-type E3s enzyme in plant immunity has not yet been uncovered, although this type of E3 ubiquitin ligase is vital to the regulation of human and animal immune signaling. ${ }^{24}$ Furthermore, it has now become evident that K63-linked polyubiquitination plays an essential role in RETINOIC ACID-INDUCIBLE GENE I (RIG-I)-like receptor (RLR)- (that is important in mammalian antiviral innate immunity) and TLR-mediated immune signaling. Such K63-linked ubiquitination has been found to be essential also to RLR- and TLR-mediated activation of the NF-kB pathway. ${ }^{25}$ Although an understanding of the role and underlying mechanistic basis of unconventional ubiquitination in plant innate immunity is still at its infant stage, ${ }^{22}$ what is clear is that the unconventional, non-degradative ubiquitination process is crucial in innate and adaptive immune responses of animals and it promises to be the same in plants (beside the already verified dominant role of conventional ubiquitination in the plant immune network).

\section{Purpose}

Returning then to the initial question of why phytotherapy can prove effective in modulating the immune responses in the animal kingdom it is possible to hypothesize that since the innate immune system is highly conserved and, in the animal kingdom, the TLRs and NOD receptors of the innate immune system share some features with PRRs and NB-LRRs receptors of the plant kingdom (which essentially has only innate immunity), the innate immune system of these two kingdoms may be sensitive to the same activators and regulatory molecules (Figure 1). Indeed, probably because some of the defense reactions directly involved in attacking invading pathogens have very ancient origins, it has been observed that both plants and animals synthesize a wide range of small antimicrobial peptides (for example, both produce an oxidative burst via conserved gp91phox NADPH oxidases) opening the way to hypothesize and identify similar ancestral mechanisms and gene shared by living organisms. ${ }^{26,27}$

Recently it has been reported that, for instance, polysaccharide PRM3 from Rhyncosia minima root enhances immune function through TLR4-NF-kB pathway. ${ }^{28}$ Thus, even if the downstream signaling pathways of pattern-recognition receptors in plants and animals are usually different, ${ }^{26}$ it could happen that the activators and regulatory molecules of plants are able to trigger the signaling pathways of the animal innate immune cells. Newly studies suggested strong similarity of defense responses associated with PTI and ETI in animals and plants and studies into animal field just recently provided some mechanistic insight into ETI in animals. ${ }^{27}$

Therefore, such as plants and different animal lineages share immune memory characteristics in innate host defense, so it could be possible that the molecules produced by the different metabolic pathways of the different compartments of a plant cell once triggered by pathogens or environmental antigens during PTI and ETI responses (i.e. reactive oxygen species, hormones, mitogen-activated protein kinase, defensive secondary metabolites called phytoalexins, salicylic acid, jasmonic acid and ethylene $)^{26,27}$ are able to evoke/activate similar defensive pathways in the innate immune system in animal kingdom. Indeed, the overall structure of microbe-associated molecule signaling pathways in plants and animals is similar and in both the kingdoms there are transmembrane LRR receptors, such as mitogenassociated protein kinase (MAPK) signaling cascades, production of active oxygen and nitrogen species, calcium fluxes, activation of transcription factors and the inducible expression of immune effectors. In particular, the components of MAPK signaling cascades form a bridge between diverse signal sensors and/or receptors and target genes in eukaryotes. ${ }^{26}$

The phytocomplexes extracted from the different organs of a plant represent the summation of the enzymes and molecules contained within each single cell. These molecules, expression of a continuous production of relevant immune effectors that could be specific or nospecific regarding the pathogen encountered (expression of sustained immune response), ${ }^{27}$ once introduced in the animal body through the phytocomplexes, arrive into a direct contact with the gut associated immune system, possibly eliciting an ETI response in mucosal innate system of animals.

Indeed, since phyto-derivatives are mainly administered through oral route, the type of immune responses that are stimulated in who uses phytotherapy have necessarily to pass through the activation of the mucosal innate immunity.

The recent discovery in different phylogenetic groups of new aspects of immunological memory also in the innate immune system ${ }^{27}$ has challenged the paradigm of differential pressure of memory of innate vs adaptive immunity: plants upon recognition of infectious microbes build immunological memory that enables a boosted defense response on re-infection. ${ }^{29}$

The action of the boosted defense effectors, therefore, is the result of concerted actions that simultaneously affects different metabolic pathways. For this reason, probably, the effects obtained by using only one or few principles extracted from the phytocomplexes are lower than those resulting from its use in full. 


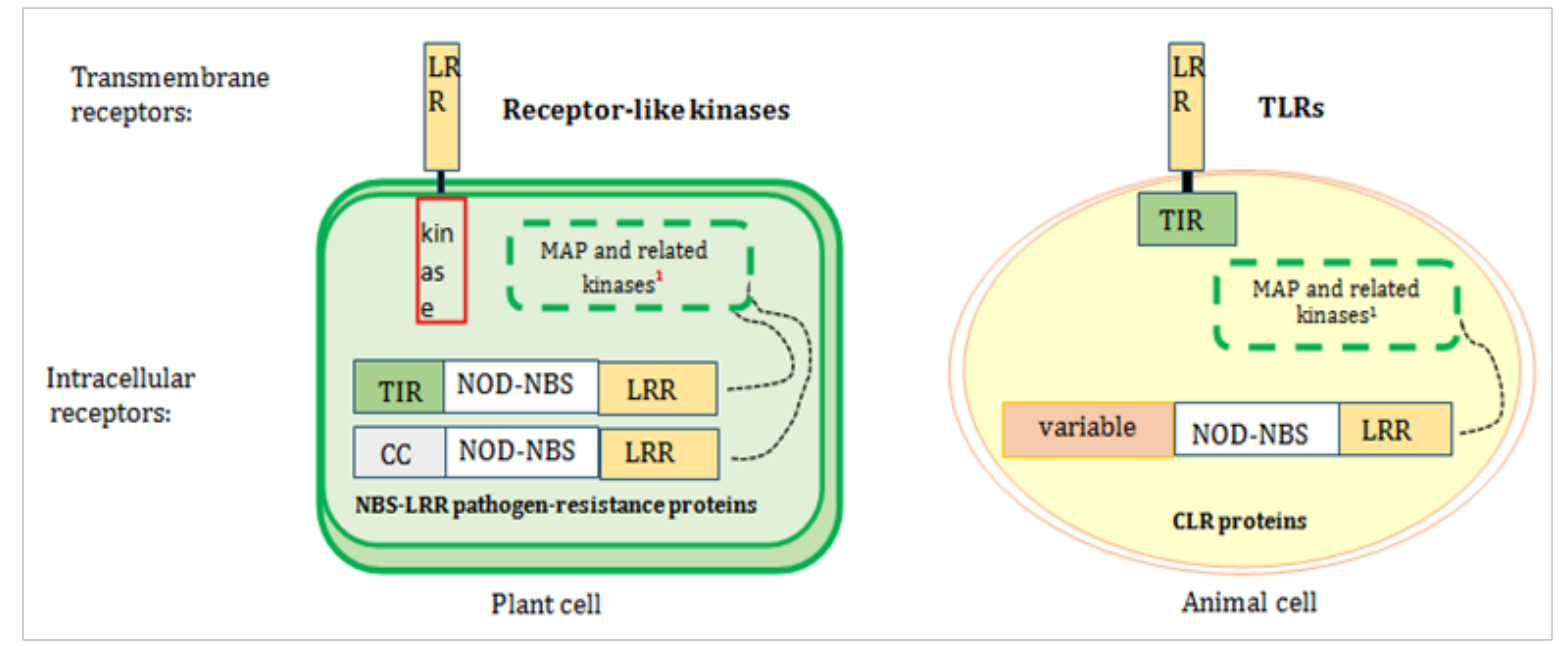

Figure I Extracellular and intracellular pattern recognition receptor (PRRs) in plants and animals. Plant receptor-like kinases and animal TLRs are similar in the structure of the transmembrane C-terminal LRRs. In both plants and animals, extracellular receptors respond to highly conserved microbe-associated molecules such as bacterial flagellin. As with receptor-like kinases and TLRs, the cytoplasmic plant NBS-LRR pathogen-resistance proteins and animal CLR proteins have the same overall tripartite structure with C-terminal LRR and central nucleotide-binding site (NBS) domains; the N-terminal domains, instead, change between plants and animals. In plants, the N-terminal domains are usually TIR or coiled-coiled domains; whereas, in animals, it could be NodI, Nod2 and NALP3. The overall conservation of the tripartite structure of animal CLR and plant NBS-LRR proteins suggests evolutionary conservation. The ubiquitary eukaryotic MAP kinases respond to environmental signals.

'The cytoplasmic kinases operate different immune responses such as generation of reactive oxygen species (ROS) and ethylene, activation of mitogen-activated protein kinase (MAPK) cascades, elevation of intracellular $\mathrm{Ca} 2+$ levels, and production and secretion of immune molecules via transcriptional reprogramming ${ }^{30}$

TIR: Toll-interleukin I receptor domain; CLR or Nod : CATERPILLER or Nod proteins;Modified by Ausubel ${ }^{26}$

\section{Conclusion}

Typically, the pathological state of a living organism can be influenced by the use of a single molecule only in a limited way, since it is always polymorphic and expression of alterations of a highly complex metabolic network. In phytotherapy, well-chosen plants do not suppress symptoms, but stimulate the homeostatic mechanisms and immune responses of the organism that, logically, needs of its biological times to heal itself. If this hypothesis were confirmed by studies aimed at evaluating the effects of phytotherapy on mucosal innate immunity ${ }^{15}$ and, possibly, innate immune memory, just as the approach to the route of administration of the vaccine has changed in the last few years, the therapeutic approach of some diseases could also be reconsidered. In fact, it would be extremely important to verify the effects of the release of phytocomplexes directly in the target sites to activate the innate immune system. Many secrets in the nature have still to be unveiled and a scientific approach to traditional medicine will warrant a more thoroughly understanding of the way of action of phytocomplexes that plants have made ready to counteract their own health problems but that probably can produce the same beneficial effects in the animal kingdom by favoring the innate and adaptive immune responses and cellular homeostasis.

\section{Acknowledgements}

None.

\section{Conflict of interest}

The author reports no conflict of interest.

\section{References}

1. Lee WC, Wung HY, Liao HH, et al. Hospital safety culture in Taiwan: a nationwide survey using Chinese version safety attitude questionnaire. BMC Health Serv Res. 2010;10(1):234-235.

2. Tauseef Sultan M, Sadiq Butt M, Nasir Qayyum MM, et al. Immunity: Plants as Effective Mediators. Crit Rev Food Sci Nutr. 2014;54(10):12981308 .

3. Colalto C. What phytotherapy needs: Evidence $\square$ based guidelines for better clinical practice. Phyt Re. 2018;32(3):413-425.

4. Joly R. Tome VI. 1st part: Du régime. In: Hippocrate, editors. Paris: Belles Lettres; 1967. 253 p.

5. Cardenas D. Let not thy food be confused with thy medicine: The Hippocratic misquotation. 2013:8(6):e260-e262.

6. Galdeano CM, Perdigo G. The Probiotic Bacterium Lactobacillus casei Induces Activation of the Gut Mucosal Immune System through Innate Immunity. Clin Vacc Immunol. 2006;13(2):219-226.

7. Bourgeon S, Raclota T, Le Mahoa Y, et al. Innate immunity, assessed by plasma NO measurements, is not suppressed during the incubation fast in eiders. Dev Comp Immunol. 2007;31(7):720-728.

8. Keil D, Luebke R, Pruett S. Quantifying the relationship between multiple immunological parameters and host resistance: probing the limits of reductionism. J Immunol. 2001;167(8):4543-4552.

9. Adamo SA. How should behavioural ecologists interpret measurements of immunity?. Anim Behav. 2004;68(6):1443-1449.

10. Watson JL, Vicario M, Wang A, et al. Immune cell activation and subsequent epithelial dysfunction by Staphylococcus enterotoxin B is attenuated by the green tea polyphenol (-)-epigallocatechin gallate. Cell 
Immunol. 2005;237(1):7-16.

11. Perdigón G, Fuller R, Raya R. Lactic Acid Bacteria and their Effect on the Immune System. Curr Issues Intest Micro. 2001;2(1):27-42.

12. Matsumoto T, Noguchi M, Hayashi O, et al. Hochuekkito, a Kampo (traditional Japanese herbal) Medicine, Enhances Mucosal IgA Antibody Response in Mice Immunized with Antigen-entrapped Biodegradable Microparticles. eCAM. 2010;7(1):69-77.

13. Iijima H, Takahashi T, Kiyono H. Mucosal immune network in the gut for the control of infectious diseases. Rev Med Virol. 2001;11(2):117-133.

14. Nochi T, Kiyono H. Innate immunity in the mucosal immune system. Curr Pharm Design. 2006;12(32):4203-4213.

15. Sonnenberg GF, Artis T. Innate lymphoid cell interactions with microbiota: implications for intestinal health and disease. Immun. 2012;37(4):601610.

16. Dwivedy A, Aich P. Importance of innate mucosal immunity and the promises it holds. Int J Gen Med. 2011;4:299-311.

17. Takeda K, Akira S. Toll-like receptors in innate immunity. Int Immunol. 2005;17(1):1-14.

18. Gururajan M, Jacob J, Pulendran B. Toll-Like Receptor Expression and Responsiveness of Distinct Murine Splenic and Mucosal B-Cell Subsets. PLoS One. 2007;2(9): e863.

19. Russell MW. Mucosal Immunology. 4th ed. In: Jones JD, Dangl JL, editors. Amsterdam: Elsevier Science; 2006;444:323-329.

20. Park E, Nedo A, Caplan JL, et al. Plant-microbe interactions: organelles and the cytoskeleton in action. New Phytol 2017;217(3):1012-1028.
21. Toruno TY, Stergiopoulos I, Coaker G. Plant-Pathogen Effectors: Cellular Probes Interfering with Plant Defenses in Spatial and Temporal Manners. Ann Rev Phytopathol. 2016;54:419-441.

22. Zhou B, Zeng L. Conventional and unconventional ubiquitination in plant immunity. Mol Plant Pathol. 2017;18(9):1313-1330.

23. Callis J. The ubiquitination machinery of the ubiquitin system, Arabidopsis Book, 2014;12,. e0174.

24. Smit JJ, Sixma TK. RBR E3-ligases at work. EMBO Reports 2014;15(2):142-154.

25. Jiang X, Chen ZJ. The role of ubiquitylation in immune defence and pathogen evasion. Nat Rev Immunol. 2011;12(1):35-48.

26. Ausubel FM. Are innate immune signaling pathways in plants and animals conserved?. Nat Immunol. 2005;6(10):973-979.

27. Gourbal B, Pinaud S, Beckers GJM, et al. Innate immune memory: An evolutionary perspective. Immunol Rev. 2018;238(1):21-40.

28. Xuejing J, Yeer L, Chao Z, et al. Polysaccharide PRM3 from Rhynchosia minima root enhances immune function through TLR4-NF- $\mathrm{B}$ pathway. BBA - General Subjects.2018;1862(8):1751-175.

29. Conrath U, Beckers GJ, Langenbach CJ, et al. Priming for enhanced defense. Annu Rev Phytopathol. 2015;53:97-119. Hye Sup Y, Jae-Hoon L, Woong June P,et al. Plant Surface Receptors Recognizing MicrobeAssociated Molecular Patterns J Plant Biol. 2018;61(3):111-120.

30. Hye Sup Y, Jae Hoon L, Woong June P, et al. Plant Surface Receptors Recognizing Microbe-Associated Molecular Patterns. J Plant Biol. 2018;61(3):111-120. 